\title{
Dynamical invariants and quantization of the one-dimensional time-dependent, damped, and driven harmonic oscillator
}

\author{
M. C. Bertin ${ }^{1}$, J. R. B. Peleteiro ${ }^{1}$, B. M. Pimentel ${ }^{2}$, and J. A. Ramirez ${ }^{1}$ \\ ${ }^{1}$ Instituto de Física, Universidade Federal da Bahia \\ ${ }^{2}$ Instituto de Física Teórica, São Paulo State University
}

\begin{abstract}
In this paper, it is proposed a quantization procedure for the one-dimensional harmonic oscillator with time-dependent frequency, time-dependent driven force, and time-dependent dissipative term. The method is based on the construction of dynamical invariants previously proposed by the authors, in which fundamental importance is given to the linear invariants of the oscillator.

Keywords: Dynamical Invariants; Quantum Damped Oscillator; Time-Dependent Systems; Dissipative Systems.

This is a pre-print of an article published in Brazilian Journal of Physics. The final authenticated version is available online at DOI: 10.1007/s13538-020-00765-8. See also online at https://rdcu.be/ b5lgb.
\end{abstract}

\section{Introduction}

Dynamical invariants were first used by Ermakov to show the connection between solutions of some special differential equations, referred to as Steen-Ermakov equations [1]. These equations were first studied by Steen [2] and then rediscovered by other authors [3]; [4]. After that, Ray and Reid used the Ermakov approach to construct invariants for a much broader class of differential equations [5]; [6]; [7]. This purely mathematical interest was the start point of significant developments in classical and quantum dynamics.

The importance of the dynamical invariants of a system should not be underrated. In classical mechanics, the dynamical constants of motion are the vari- 
ables that allow complete integration of dynamical systems. In classical field theories, symmetries of lagrangian systems are related to continuity equations and time-invariants through the Noether theorem [8] In quantum field theory, Casimir invariants of symmetry groups are essential to the understanding of the fundamental particle structure of our universe [9].

In quantum mechanics, a complete characterization of a quantum system is achieved by the knowledge of a complete set of time-invariant observables, which are also generators of a complete symmetry of the system. The process of quantization, therefore, is accomplished by finding an invariant set of stationary eigenvectors which generates, hopefully, a Hilbert space. Symmetries are linked to invariants, and invariants are linked to the very existence of quantum states, on a very fundamental level.

In time-dependent systems, dynamical invariants play a major role, since the energy is no longer conserved, and sometimes even defined. Particularly, in quantum mechanics, systems with time-dependent Hamiltonians do not have well-defined energy spectra. Even in the case where a complete basis of eigenvectors exists, one cannot be sure that this condition persists in time. When quantization is allowed, the problem of time-dependent hamiltonians can be dealt with by finding a hermitian quadratic invariant, for which the eigenvalue problem is well defined [10]. Time-dependent systems appear in several applications in physics such as ion traps [11]; [12]; [13], optical cavities [14], and to perform algorithms in quantum computation [15]; [16].

There are several methods to calculate dynamical invariants. In the classical case, we have Lutzky's approach [17]; [18], which consists of the application of the Noether theorem. Another method is the dynamical algebra approach [19]; [20]. Recently, the authors developed a new way to calculate dynamical invariants [21], which consists of combinations of the equations of motion. These last two methods can be used in both, classical and quantum cases.

In this work, we show how the definition of first-order invariants allows us to approach the quantization of the one-dimension time-dependent, damped, driven harmonic oscillator (TDDDHO). In sec. 2, we follow [21] and calculate the linear invariants for the TDDDHO by taking the combinations of the equations of motion. Next, in sec. 3, we construct the quadratic invariant and find a SteenErmakov-like equation. In sec. 4, we perform the quantization of the TDDDHO using the algebra of the first-order invariants. Sec. 5 presents the coordinate representation in the form of wave eigenfunctions of the quadratic invariant, 
along with a general expression for the uncertainty relations between the observables $(q, p)$. In section 6 , we address the problem of the dissipative oscillator with constant parameters and general driven force. Finally, in sec. 7 , we present our main observations.

\section{First-order invariants of the oscillator}

Let us start with the hamiltonian operator

$$
H=\frac{1}{2 m} e^{-G(t)} p^{2}+\frac{1}{2} m \omega^{2}(t) e^{G(t)} q^{2}-e^{G(t)} F(t) q
$$

in which the canonical pair $(q, p)$ are Hilbert space operators with commutation relations $[q, p]=i \hbar \mathbf{1},[q, q]=0$, and $[p, p]=0$. The term $\omega(t)$ represents a time dependent angular frequency, $F(t)$ stands for a time dependent driven force, and $G(t)$ is another time dependent function. These functions are supposed to be at least of class $C^{2}$. This operator can be seen as a generalization of the BatemanCaldirola-Kanai (BCK) model for the dissipative harmonic oscillator [22]; [23]; [24].

Heisenberg's equations for the hamiltonian (1) are given by

$$
\begin{aligned}
\dot{q} & =e^{-G} p / m \\
\dot{p} & =e^{G} F-e^{G} m \omega^{2} q, \\
\ddot{q} & =\frac{1}{m} F-2 g \dot{q}-\omega^{2} q, \quad g(t) \equiv \frac{1}{2} \dot{G}(t) .
\end{aligned}
$$

The function $g(t)$ has the interpretation of a dissipative term.

We proceed by calculating the first-order dynamical invariants related to (2) with the method proposed by [21]. In this case we define two arbitrary complex functions $\alpha(t)$ and $\beta(t)$. Multiplying (2a) by $\alpha$ and (2b) by $\beta$, building the linear combination, and isolating the total time derivative results in the expression

$$
\frac{d}{d t}(\beta p+\alpha m q)=\left(\alpha e^{-G}+\dot{\beta}\right) p+m\left(\dot{\alpha}-e^{G} \omega^{2} \beta\right) q+\beta e^{G} F
$$


Now, we define the function

$$
\mathcal{F}(\beta, t) \equiv \int_{t_{0}}^{t} e^{G(\tau)} \beta(\tau) F(\tau) d \tau, \quad \beta\left(t_{0}\right) F\left(t_{0}\right)=0,
$$

for which we have the identity

$$
\beta e^{G} F=\frac{d \mathcal{F}}{d t}
$$

With (5), we may express (3) in the form

$$
\frac{d}{d t}(\beta p+\alpha m q-\mathcal{F})=\left(\alpha e^{-G}+\dot{\beta}\right) p+m\left(\dot{\alpha}-e^{G} \omega^{2} \beta\right) q
$$

If the parameters $\alpha$ and $\beta$ satisfy the ODEs

$$
\begin{aligned}
\alpha+e^{G} \frac{d \beta}{d t} & =0, \\
\frac{d \alpha}{d t}-e^{G} \omega^{2} \beta & =0,
\end{aligned}
$$

the polynomial

$$
I=\beta p+\alpha m q-\mathcal{F}(\beta, t)
$$

becomes a first-order invariant of the system (2).

The functions $\alpha$ and $\beta$ are not independent solutions, therefore, we may write (6) depending on $\beta$ alone:

$$
I=\beta p-m e^{G} \dot{\beta} q-\mathcal{F},
$$

where $\beta$ is now a solution of the equation

$$
\ddot{\beta}+2 g \dot{\beta}+\omega^{2} \beta=0 .
$$

Now we suppose there is a solution of (8) with the form 


$$
\beta \equiv \rho(t) e^{i \phi(t)}
$$

with $\phi(t)$ and $\rho(t)$ both real functions. Eq. (8) then becomes

$$
\begin{array}{r}
\ddot{\rho}+2 g \dot{\rho}+\left(\omega^{2}-\dot{\phi}^{2}\right) \rho=0, \\
2 \dot{\rho} \dot{\phi}+\rho(\ddot{\phi}+2 g \dot{\phi})=0,
\end{array}
$$

and, in this case, it is straightforward to show that

$$
\beta^{*}=\rho(t) e^{-i \phi(t)}
$$

is also a solution of (8). Therefore, the operator

$$
I^{\dagger}=\beta^{*} p-m e^{G} \dot{\beta}^{*} q-\mathcal{F}^{*}
$$

is also a linearly independent first-order dynamical invariant.

\section{The second-order invariant of the oscillator}

We may also build quadratic invariants from the equations of motion (2). Without the driving force, it would be sufficient to build linear combinations of products of these equations. However, this is not the case when the driving force is in place. Let us observe the following products between (2a) and (2b):

$$
\begin{aligned}
\frac{d q^{2}}{d t} & =\frac{e^{-G}}{m}\{q, p\}, \\
\frac{d}{d t}\{q, p\} & =2 \frac{e^{-G}}{m} p^{2}+2 e^{G} F q-2 e^{G} m \omega^{2} q^{2}, \\
\frac{d p^{2}}{d t} & =2 e^{G} F p-e^{G} m \omega^{2}\{q, p\},
\end{aligned}
$$

where $\{q, p\} \equiv q p+p q$ represents the anti-commutator. The r.h.s. of these equations fail to be purely quadratic forms, because of the presence of the driving 
force. This situation is corrected with the use of the equations of motion (2) themselves.

Now we take a set of time-dependent functions $c_{i}=\left(c_{1}, c_{2}, c_{3}, c_{4}, c_{5}\right)$, build a linear combination of (2) and (10), and collect the total time derivative. The result is given by

$$
\begin{aligned}
\frac{d}{d t}\left[c_{1} \frac{q^{2}}{2}+\right. & \left.\frac{1}{2} c_{2}\{q, p\}+c_{3} \frac{p^{2}}{2}+c_{4} q+c_{5} p-\mathcal{F}\left(c_{5}, t\right)\right]= \\
= & \left(\frac{1}{2} \frac{d c_{3}}{d t}+c_{2} \frac{e^{-G}}{m}\right) p^{2}+\left(\frac{1}{2} \frac{d c_{1}}{d t}-c_{2} e^{G} m \omega^{2}\right) q^{2}+ \\
& +\frac{1}{2}\left(\frac{d c_{2}}{d t}+c_{1} \frac{e^{-G}}{m}-c_{3} e^{G} m \omega^{2}\right)\{q, p\}+ \\
& +\left(c_{2} e^{G} F-c_{5} e^{G} m \omega^{2}+\frac{d c_{4}}{d t}\right) q+ \\
& +\left(\frac{d c_{5}}{d t}+c_{4} \frac{e^{-G}}{m}+c_{3} e^{G} F\right) p
\end{aligned}
$$

Hence, the second-order polynomial

$$
I_{Q}=\frac{c_{1}}{2} q^{2}+\frac{c_{2}}{2}\{q, p\}+\frac{c_{3}}{2} p^{2}+c_{4} q+c_{5} p-\mathcal{F}\left(c_{5}, t\right)
$$

is a dynamical invariant if the equations

$$
\begin{aligned}
\frac{d c_{3}}{d t}+2 \frac{e^{-G}}{m} c_{2} & =0, \\
\frac{d c_{1}}{d t}-2 c_{2} e^{G} m \omega^{2} & =0, \\
\frac{d c_{2}}{d t}+c_{1} \frac{e^{-G}}{m}-c_{3} e^{G} m \omega^{2} & =0, \\
\frac{d c_{4}}{d t}+c_{2} e^{G} F-c_{5} e^{G} m \omega^{2} & =0, \\
\frac{d c_{5}}{d t}+c_{3} e^{G} F+c_{4} \frac{e^{-G}}{m} & =0,
\end{aligned}
$$

are satisfied.

We notice that (11) can be rewritten to depend only on the functions $c_{3}$ and $c_{5}$. Let us rename them as $\gamma$ and $\sigma$, respectively. In this case, the second-order 
invariant is given by

$$
\begin{aligned}
I_{Q}= & \frac{1}{2}\left(m e^{G}\right)^{2}\left(\frac{1}{2} \frac{d^{2} \gamma}{d t^{2}}+g \frac{d \gamma}{d t}+\omega^{2} \gamma\right) q^{2}-\frac{m}{4} e^{G} \frac{d \gamma}{d t}\{q, p\} \\
& +\frac{\gamma}{2} p^{2}-m e^{G}\left(\frac{d \sigma}{d t}+\gamma e^{G} F\right) q+\sigma p-\mathcal{F}(\sigma, t),
\end{aligned}
$$

and ODEs for $\gamma$ and $\sigma$ follow:

$$
\begin{array}{r}
\frac{1}{2} \frac{d^{3} \gamma}{d t^{3}}+3 g \frac{d^{2} \gamma}{d t^{2}}+\left(\dot{g}+4 g^{2}+2 \omega^{2}\right) \frac{d \gamma}{d t}+\left(\frac{d \omega^{2}}{d t}+4 \omega^{2} g\right) \gamma=0 \\
\frac{d^{2} \sigma}{d t^{2}}+2 g \frac{d \sigma}{d t}+\omega^{2} \sigma=-\frac{3}{2} e^{G} F \frac{d \gamma}{d t}-e^{G}\left(\frac{d F}{d t}+4 g F\right) \gamma
\end{array}
$$

Eq. (13a) above has a first integral given by

$$
\frac{d^{2} \gamma}{d t^{2}}+2 g \frac{d \gamma}{d t}+2 \omega^{2} \gamma=\frac{1}{2 \gamma}\left(\frac{d \gamma}{d t}\right)^{2}+e^{-2 G} C,
$$

which can be turned into a Steen-Ermakov-like equation through the change of variables $\gamma=r^{2}$ :

$$
\frac{d^{2} r}{d t^{2}}+2 g r^{3} \frac{d r}{d t}+\omega^{2} r=\frac{e^{-2 G} C}{2 r^{3}}
$$

The Steen-Ermakov equation itself is obtained when $g=0$. The equation for $\sigma$ is relevant only if the force term is present. Otherwise, the above invariants resemble the case of the oscillator with time-dependent frequency already addressed in the ref. [21].

\section{Quantization of the oscillator}

Now we wish to explore the fact that the first-order operators (7) and (9) are two dynamical invariants of the oscillator if $\beta$ and $\beta^{*}$ are two L.I. solutions of (8). The commutation relations are found to be 


$$
\left[I, I^{\dagger}\right]=\Omega \mathbf{1}, \quad \Omega \equiv i m \hbar e^{G} W, \text { and } \quad W \equiv \dot{\beta}^{*} \beta-\beta^{*} \dot{\beta}
$$

The remaining relations are just $[I, I]=\left[I^{\dagger}, I^{\dagger}\right]=0$. In fact, using (8) it is straightforward to see $\Omega$ is a constant of motion by itself.

We define the operators

$$
a \equiv \frac{I}{\sqrt{\Omega}}, \quad a^{\dagger} \equiv \frac{I^{\dagger}}{\sqrt{\Omega}},
$$

which obey the commutation relations

$$
\left[a, a^{\dagger}\right]=\mathbf{1}, \quad[a, a]=\left[a^{\dagger}, a^{\dagger}\right]=0 .
$$

Since $a$ and $a^{\dagger}$ are invariants, any product between them is also a dynamical invariant. This fact allows the introduction of the number operator

$$
\hat{\boldsymbol{n}} \equiv a^{\dagger} a,
$$

which is a time-conserved self-adjoint quadratic quantity. The quantization is performed by assuming the existence of a complete set of eigenstates $|n\rangle$, i.e.,

$$
\hat{\boldsymbol{n}}|n\rangle=n|n\rangle,
$$

where $n$ is a positive real number, because of the positivity of the inner product. The complete algebra of the oscillator is shown to be given by

$$
\left[a, a^{\dagger}\right]=\mathbf{1}, \quad[\hat{\boldsymbol{n}}, a]=-a, \quad\left[\hat{\boldsymbol{n}}, a^{\dagger}\right]=a^{\dagger},
$$

from where we derive

$$
a|n\rangle=\sqrt{n}|n-1\rangle, \quad a^{\dagger}|n\rangle=\sqrt{n+1}|n+1\rangle,
$$

therefore, $a$ and $a^{\dagger}$ are ladder operators. As usual, we suppose the existence of a fundamental state, defined by $a|0\rangle=0$, and therefore $n$ must be a natural 
number. All other eigenstates can be derived from

$$
|n\rangle=\frac{\left(a^{\dagger}\right)^{n}}{\sqrt{n !}}|0\rangle,
$$

and the quantization procedure is complete.

We see that the dynamical algebra of the operators $\hat{\boldsymbol{n}}, a$, and $a^{\dagger}$ is the same as of the simple harmonic oscillator, so it is the Hilbert space spanned by the $|n\rangle$ states. What is distinct among the several possible choices of the parameters $(g, \omega, \mathcal{F})$ are the behavior of the physical characteristic functions of the model, as the energy values, expected values, and others.

\section{Eigenvalue solutions, eigenfunctions, and uncer- tainty}

Let us now show the explicit form of the number operator:

$$
\hat{\boldsymbol{n}}=a^{\dagger} a=\frac{1}{\Omega}\left(\frac{1}{2}\left[I^{\dagger}, I\right]+\frac{1}{2}\left\{I^{\dagger}, I\right\}\right)=\frac{1}{2}\left(\frac{1}{\Omega}\left\{I^{\dagger}, I\right\}-\mathbf{1}\right) .
$$

The quantity $\frac{1}{2}\left\{I^{\dagger}, I\right\}$ is also a quadratic self-adjoint dynamical invariant, calculated by

$$
\begin{aligned}
\frac{1}{2}\left\{I^{\dagger}, I\right\}= & \beta^{*} \beta p^{2}-\frac{1}{2} m e^{G}\left(\beta^{*} \dot{\beta}+\dot{\beta}^{*} \beta\right)\{q, p\}+m^{2} e^{2 G} \dot{\beta}^{*} \dot{\beta} q^{2} \\
& -\left(\beta^{*} \mathcal{F}+\mathcal{F}^{*} \beta\right) p+m e^{G}\left(\dot{\beta}^{*} \mathcal{F}+\dot{\beta} \mathcal{F}^{*}\right) q+\frac{1}{2} \mathcal{F}^{*} \mathcal{F}
\end{aligned}
$$

The definition of the real function $\gamma \equiv 2 \beta^{*} \beta$ results in the expression

$$
\begin{aligned}
\frac{1}{2}\left\{I^{\dagger}, I\right\}= & \frac{1}{2}\left(m e^{G}\right)^{2}\left(\frac{1}{2} \frac{d^{2} \gamma}{d t^{2}}+g \frac{d \gamma}{d t}+\omega^{2} \gamma\right) q^{2}-\frac{1}{4} m e^{G} \frac{d \gamma}{d t}\{q, p\}+\frac{1}{2} \gamma p^{2} \\
& -\left(\beta^{*} \mathcal{F}+\mathcal{F}^{*} \beta\right) p+m e^{G}\left(\dot{\beta}^{*} \mathcal{F}+\mathcal{F}^{*} \dot{\beta}\right) q+\frac{1}{2} \mathcal{F}^{*} \mathcal{F}
\end{aligned}
$$

Now, we define $\sigma \equiv-\beta^{*} \mathcal{F}-\mathcal{F}^{*} \beta$, which leads to 


$$
\dot{\beta}^{*} \mathcal{F}+\mathcal{F}^{*} \dot{\beta}=-\left(\frac{d \sigma}{d t}+\gamma e^{G} F\right)
$$

On the other hand, $\mathcal{F}^{*} \mathcal{F}=\mathcal{F}\left(\beta^{*}, t\right) \mathcal{F}(\beta, t)=-2 \mathcal{F}(\sigma, t)$. Therefore,

$$
\begin{aligned}
\frac{1}{2}\left\{I^{\dagger}, I\right\}= & \frac{1}{2}\left(m e^{G}\right)^{2}\left(\frac{1}{2} \frac{d^{2} \gamma}{d t^{2}}+g \frac{d \gamma}{d t}+\omega^{2} \gamma\right) q^{2}-\frac{1}{4} m e^{G} \frac{d \gamma}{d t}\{q, p\}+\frac{1}{2} \gamma p^{2} \\
& -m e^{G}\left(\frac{d \sigma}{d t}+\gamma e^{G} F\right) q+\sigma p-\mathcal{F}(\sigma, t)
\end{aligned}
$$

which is precisely the second-order invariant (12). The above result implies

$$
I_{Q}=\Omega\left(\hat{\boldsymbol{n}}+\frac{1}{2}\right)
$$

so $I_{Q}$ has the same eigenstates of $\hat{\boldsymbol{n}}$.

Moreover, considering $\langle q|a| 0\rangle=0$, and the eigenvalue problem $q\left|q^{\prime}\right\rangle=q^{\prime}\left|q^{\prime}\right\rangle$, the eigenfunction of the fundamental state obeys the equation

$$
\left(\mathcal{F}+m e^{G} \dot{\beta} q+i \hbar \beta \frac{d}{d q}\right) \psi_{0}(q)=0
$$

which has the solution

$$
\psi_{0}=A \exp \left[-\frac{1}{2} \frac{1}{i \hbar \beta}\left(m e^{G} \dot{\beta} q^{2}+2 \mathcal{F} q\right)\right]
$$

with the normalization constant

$$
A=\left(\frac{1}{2 \pi \hbar^{2}} \frac{\Omega}{\beta^{*} \beta}\right)^{1 / 4} \exp \left[-\left(\frac{1}{\beta^{*} \beta}\right)^{2} \frac{1}{\Omega}\left(\operatorname{Im}\left(\beta^{*} \mathcal{F}\right)\right)^{2}\right]
$$

The complete set of normalized eigenfunctions are found to be

$$
\psi_{n}=\frac{1}{\sqrt{2^{n} \cdot n !}}\left(i \sqrt{\frac{\beta^{*}}{\beta}}\right)^{n} \psi_{0} H_{n}\left[\sqrt{\frac{\Omega}{2 \beta^{*} \beta}}\left(\frac{q}{\hbar}+\frac{2}{\Omega} \operatorname{Im}\left(\beta^{*} \mathcal{F}\right)\right)\right],
$$

where $H_{n}(x)$ are the Hermite polynomials. Here, we stress the fact that (23) are eigenfunctions of the operator $I_{Q}$, but they are also solutions of the Schrödinger 
equation $\left(i \hbar \partial_{t}-H\right) \psi=0$. These states are the same found in [25], where coherent states of the general one-dimensional oscillator are discussed.

Writing the canonical variables in the form

$$
\begin{aligned}
& q=\frac{i \hbar}{\sqrt{\Omega}}\left(\beta^{*} a-\beta a^{\dagger}\right)-2 \frac{\hbar}{\Omega} \operatorname{Im}\left(\beta^{*} \mathcal{F}\right), \\
& p=\frac{i m \hbar e^{G}}{\sqrt{\Omega}}\left(\dot{\beta}^{*} a-\dot{\beta} a^{\dagger}\right)-\frac{2 m \hbar e^{G}}{\Omega} \operatorname{Im}\left(\dot{\beta}^{*} \mathcal{F}\right),
\end{aligned}
$$

allows us to calculate the uncertainty relations

$$
(\Delta q)_{n}^{2}(\Delta p)_{n}^{2}=\frac{2 m^{2} \hbar^{4} e^{2 G}}{\Omega^{2}} \gamma \dot{\beta}^{*} \dot{\beta}\left(n+\frac{1}{2}\right)^{2}
$$

\section{The underdamping oscillator}

Let us analyze the case $g^{2} \leq \omega^{2}$ with both $\omega$ and $g$ constant parameters, and $F=$ $F(t)$ still arbitrary. In this case, the function $G$ should be linear in $t$. Let us suppose it to have the form of $G=2 g t$. We also have the solution

$$
\beta=\exp (-g t) \exp (i \bar{\omega} t), \quad \bar{\omega}^{2} \equiv \omega^{2}-g^{2},
$$

while $\beta^{*}$ is just the complex conjugate. With (24), the linear dynamical invariants of the system become

$$
I=e^{i \bar{\omega} t}\left[e^{-g t} p+m(g-i \bar{\omega}) e^{g t} q-e^{-i \bar{\omega} t} \mathcal{F}\right]
$$

together with the adjoint operator $I^{\dagger}$. We also have the function $\Omega=2 m \bar{\omega} \hbar$, which gives the ladder operators

$$
a=\frac{e^{i \bar{\omega} t}}{\sqrt{2 m \bar{\omega} \hbar}}\left[e^{-g t} p+m(g-i \bar{\omega}) e^{g t} q-e^{-i \bar{\omega} t} \mathcal{F}\right]
$$

and the adjoint $a^{\dagger}$.

In this case, the quadratic invariant can be found from (12): 


$$
\begin{aligned}
I_{Q}= & e^{-2 g t} p^{2}+m^{2} \omega^{2} e^{2 g t} q^{2}+m g\{q, p\} \\
& -m e^{G}\left(\frac{d \sigma}{d t}+\gamma e^{G} F\right) q+\sigma p-\mathcal{F}(\sigma, t) .
\end{aligned}
$$

Note that $I_{Q}$ is an invariant observable, so the invariant eigenvalues

$$
I_{n}=2 m \bar{\omega} \hbar\left(n+\frac{1}{2}\right)
$$

represent invariant characteristic values of the oscillator.

It is possible to calculate the fundamental eigenfunction with the use of (22), resulting in the normalized function

$$
\begin{aligned}
\psi_{0}= & e^{g t / 2}\left(\frac{m \bar{\omega}}{\pi \hbar}\right)^{1 / 4} \exp \left[-\frac{1}{2 m \hbar \bar{\omega}} e^{2 g t}\left(\operatorname{Im}\left(e^{-i \bar{\omega} t} \mathcal{F}\right)\right)^{2}\right] \times \\
& \times \exp \left(-\frac{i}{2} \frac{m \bar{g}}{\hbar} e^{2 g t} q^{2}+\frac{i}{\hbar} e^{-i \bar{\omega} t} \mathcal{F} e^{g t} q\right)
\end{aligned}
$$

where $\bar{g} \equiv g-i \bar{\omega}$. A straightforward calculation shows that

$$
\psi_{n}=\frac{(i)^{n}}{\sqrt{2^{n} \cdot n !}} e^{-i n \bar{\omega} t} \psi_{0} H_{n}(x)
$$

are the normalized eigenfunctions, where

$$
x=\sqrt{\frac{m \bar{\omega}}{\hbar}} e^{g t} q-\sqrt{\frac{1}{m \hbar \bar{\omega}}} \operatorname{Im}\left(e^{-i \bar{\omega} t} \mathcal{F}\right) .
$$

Moreover, we have the uncertainty relations

$$
(\Delta q)_{n}^{2}(\Delta p)_{n}^{2}=\hbar^{2} \frac{\bar{\omega}^{2}-g^{2}}{\bar{\omega}^{2}}\left(n+\frac{1}{2}\right)^{2}
$$

which are time-independent.

Let us display some results for the case $F=F_{0} \sin (\alpha t)$. Since the force term does not change the differential equations for $\beta$ and $\beta^{*}$, their solutions are the 
same as the ones proposed in this section. Now that we have a force term we need to calculate $\mathcal{F}$ given by (4), resulting

$$
\mathcal{F}=F_{0} e^{\bar{g}^{*} t} \frac{\bar{g}^{*} \sin (\alpha t)-\alpha \cos (\alpha t)+\alpha e^{-\bar{g}^{*} t}}{\left(\bar{g}^{*}\right)^{2}+\alpha^{2}} .
$$

The parameter $\sigma$ is given by

$$
\begin{aligned}
\sigma= & \frac{-2 F_{0}}{\left[\left(g^{2}-\bar{\omega}^{2}+\alpha^{2}\right)^{2}+4 \bar{\omega}^{2} g^{2}\right]}\left\{2 \bar{\omega} g\left[\bar{\omega} \sin (\alpha t)-\alpha e^{-g t} \sin (\bar{\omega} t)\right]\right. \\
& \left.+\left(g^{2}-\bar{\omega}^{2}+\alpha^{2}\right)\left[g \sin (\alpha t)-\alpha \cos (\alpha t)+\alpha e^{-g t} \cos (\bar{\omega} t)\right]\right\}
\end{aligned}
$$

and $\mathcal{F}(\sigma, t)$ becomes

$$
\begin{aligned}
\mathcal{F}(\sigma, t)= & \frac{-2 F_{0}^{2} e^{2 g t}}{\left[\left(g^{2}-\bar{\omega}^{2}+\alpha^{2}\right)^{2}+4 \bar{\omega}^{2} g^{2}\right]}\left\{\left[\bar{\omega} \sin (\alpha t)-\alpha e^{-g t} \sin (\bar{\omega} t)\right]^{2}\right. \\
& \left.+\left[g \sin (\alpha t)-\alpha \cos (\alpha t)+\alpha e^{-g t} \cos (\bar{\omega} t)\right]^{2}\right\}
\end{aligned}
$$

The simple harmonic oscillator is trivially recovered in the case $G=0, \bar{\omega}=\omega$, and $\mathcal{F}=0$, which also gives the condition $\sigma=0$.

\section{$7 \quad$ Further observations}

In this work, we showed a procedure for the quantization of the harmonic oscillator with time-dependent frequency, time-dependent driven force, and timedependent dissipative term. The procedure is based on the construction of the linear invariants of the BCK Hamiltonian (1), which turns out to be ladder operators. We also construct the Hilbert space of the system and calculate the wave eigenfunctions.

This approach shows that the fundamental quantities turn out to be the linear invariants. Other attempts of analyzing the quantum oscillator from the dynamical invariant point of view can be found in the literature, most of them are based on the second-order invariant (16) as the proper Hamiltonian operator, as the case of [26]. However, the fundamental role of the linear invariants for the 
quantization of the oscillators can be found in [27]; [25]. In fact, the procedure of the ref. [25] is very close to the one employed here. In the case of the underdamped oscillator, we also report to the refs. [28], where the authors propose a quantization procedure based on the construction of first-order actions, and also to the ref. [29].

We found that the abstract Hilbert space of the general quadratic oscillator is the same as the simple harmonic oscillator. However, it is not a surprise that the same is not observed with the solutions of the Schrödinger equation, which are also eigenfunctions of the quadratic invariant $I_{Q}$. The wave functions $\psi_{n}$ are time-dependent and lead, in the general case, to time-dependent expectation values and uncertainty relations for the canonical operators. In the special case of constant parameters, however, the uncertainty relations between $q$ and $p$ are time-independent.

We note that the procedure in [21] does not need a Hamiltonian function, but can be implemented from the equation of motion (2c). However, some caution would be advised. First, the first-order equations,

$$
\dot{x}=y, \quad \dot{y}=-\dot{G} y-\omega^{2} x+F / m,
$$

do not constitute a canonical system, since it is not compatible with the condition $[x, y]=i \hbar$. A direct calculation shows that

$$
\frac{d}{d t}[x, y]=-\dot{G}[x, y], \quad \Longrightarrow \quad[x, y]=i \hbar \exp (-\dot{G} t)
$$

This result alone would make us believe that the system is indeed dissipative since it is clear that the allowed classical states would collapse to the zero volume in time. However, if there would be a local transformation to a set of canonical variables, a volume preserved phase-space would emerge. This phase-space would obey the Darboux and the Liouville theorems. The condition for the existence of such transformation is given by $\{x, y\}=e^{-\dot{G} t}$, where $\{\bullet, \bullet\}$ are the Poisson brackets with respect to the variables $(q, p)$. This condition is indeed quite general. However, the only allowed transformation that leads to the two first equations of (2a) is given by $x=q$, and $y=e^{-\dot{G} t / 2} p$ provided $G$ is homogeneous of degree zero. Both sets of first-order equations are not generally compatible. 


\section{Acknowledgments}

This study was financed in part by the Coordenação de Aperfeiçoamento de Pessoal de Nível Superior - Brasil (CAPES) - Finance Code 001. B. M. Pimentel thanks the Conselho Nacional de Desenvolvimento Científico e Tecnológico (CNPq) for partial support.

\section{References}

1. P. Ermakov, Applicable Analysis and Discrete Mathematics 2, 123 (2008).

2. R. Redheffer, Aequationes Mathematicae 61, 131 (2001).

3. W. E. Milne, Physical Review 35, 863 (1930).

4. E. Pinney, Proceedings of the American Mathematical Society 1, 681 (1950).

5. J. R. Ray and J. L. Reid, Physics Letters A 71, 317 (1979).

6. J. R. Ray and J. L. Reid, Physics Letters A 74, 23 (1979).

7. J. R. Ray, Physics Letters A 78, 4 (1980).

8. D. E. Neuenschwander, Emmy Noether's Wonderful Theorem (Johns Hopkins University Press, 2011).

9. E. Wigner, The Annals of Mathematics 40, 149 (1939).

10. H. R. Lewis and W. B. Riesenfeld, Journal of Mathematical Physics 10, 1458 (1969).

11. W. Paul, Reviews of Modern Physics 62, 531 (1990).

12. D. Leibfried, R. Blatt, C. Monroe, and D. Wineland, Reviews of Modern Physics 75, 281 (2003).

13. E. Torrontegui, S. Ibáñez, X. Chen, A. Ruschhaupt, D. Guéry-Odelin, and J. G. Muga, Physical Review A 83, (2011).

14. H. Johnston and S. Sarkar, Journal of Physics A: Mathematical and General 29, 1741 (1996).

15. M. S. Sarandy, E. I. Duzzioni, and R. M. Serra, Physics Letters A 375, 3343 (2011).

16. U. Güngördü, Y. Wan, M. A. Fasihi, and M. Nakahara, Physical Review A 86, (2012). 
17. M. Lutzky, Journal of Physics A: Mathematical and General 11, 249 (1978).

18. M. Lutzky, Physics Letters A 68, 3 (1978).

19. H. J. Korsch, Physics Letters A 74, 294 (1979).

20. R. S. Kaushal and H. J. Korsch, Journal of Mathematical Physics 22, 1904 (1981).

21. M. C. Bertin, B. M. Pimentel, and J. A. Ramirez, Journal of Mathematical Physics 53, 042104 (2012).

22. H. Bateman, Physical Review 38, 815 (1931).

23. P. Caldirola, Il Nuovo Cimento 18, 393 (1941).

24. E. Kanai, Progress of Theoretical Physics 3, 440 (1948).

25. V. V. Dodonov and V. I. Man'ko, Physical Review A 20, 550 (1979).

26. H. R. Lewis, Physical Review Letters 18, 636 (1967).

27. I. A. Malkin and V. I. Man'ko, Physics Letters A 32, 243 (1970).

28. D. M. Gitman and V. G. Kupriyanov, The European Physical Journal C 50, 691 (2007).

29. M. C. Baldiotti, R. Fresneda, and D. M. Gitman, Physics Letters A 375, 1630 (2011). 